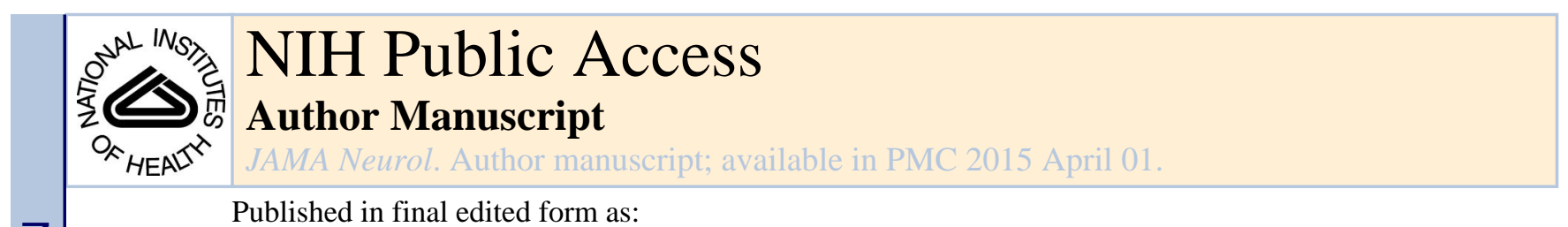

Published in final edited form as:

JAMA Neurol. 2014 October ; 71(10): 1209-1210. doi:10.1001/jamaneurol.2014.1193.

\title{
Lyme Disease:
}

\section{Authentic Imitator or Wishful Imitation?}

Michael T. Melia, MD,

Division of Infectious Diseases, Department of Medicine, Johns Hopkins University School of Medicine, Baltimore, Maryland

Paul M. Lantos, MD, and

Department of Internal Medicine, Duke University School of Medicine, Durham, North Carolina; and Department of Pediatrics, Duke University School of Medicine, Durham, North Carolina

\section{Paul G. Auwaerter, MD}

Division of Infectious Diseases, Department of Medicine, Johns Hopkins University School of Medicine, Baltimore, Maryland

The spirochete Borrelia burgdorferi may afflict skin, heart, joints, and the central or peripheral nervous system. This agent of Lyme disease, perhaps because of its varied presentations, is often raised as the cause of headache, fatigue, and subjective neurocognitive dysfunction. For clinicians who trained in the 20th century when the spirochete Treponema pallidum was invoked as the "Great Imitator," testing for Lyme disease now seems as or more common than it had been for syphilis. With a narrower disease spectrum than syphilis, is such frequent testing for Lyme disease justified, and how should results be interpreted?

If contemplating Lyme neuroborreliosis, understanding the epidemiologic likelihood of acquiring this tick-borne infection, its potential manifestations, and proper interpretation of serologic testing are all essential. For patients from Lyme-endemic areas, Lyme disease is among the most common causes of facial nerve palsy, aseptic meningitis, and neuroradiculitis. While such manifestations of early disseminated Lyme disease arise in up to $10 \%$ to $15 \%$ of patients not treated at an earlier stage, late neurologic complications such as encephalitis and diffuse polyneuropathy are exceptionally rare. Despite the rarity of late Lyme neuroborreliosis, many physicians include Lyme disease in their differential diagnosis for a variety of chronic neurologic syndromes. This approach may be partly because of brain imaging reports that commonly reference Lyme disease among the possible explanations for nonspecific white matter changes, despite the fact that $B$ burgdorferi infection essentially never causes such findings. ${ }^{1}$

Copyright 2014 American Medical Association. All rights reserved.

Corresponding Author: Michael T. Melia, MD, Johns Hopkins University School of Medicine, 1830 E Monument St, Ste 448, Baltimore, MD 21287 (mmelia4@jhmi.edu).

Conflict of Interest Disclosures: None reported.

Disclaimer: The content is solely the responsibility of the authors and does not necessarily represent the official views of the National Institutes of Health. 
$B$ burgdorferi infection is well known to affect the seventh and less commonly sixth cranial nerves. An often-posed question, therefore, is whether B burgdorferi infection can produce other isolated cranial neuropathies, such as sensorineural hearing loss and optic neuritis. A recent review advocated against screening patients with sudden-onset sensorineural hearing loss for Lyme disease because of an unproven causative relationship. ${ }^{2}$ While children with Lyme meningitis can have papilledema, and adults with $B$ burgdorferi-driven optic neuritis manifesting as papillitis have been described, this latter phenomenon appears uncommon. A case series of 440 patients with optic neuritis from a Lyme-endemic region found 25 seropositive but only 1 with evidence of active $B$ burgdorferi infection. ${ }^{3}$ The rarity of this association was reinforced in the accompanying literature review.

Lyme disease is often investigated during the initial evaluation of conditions such as multiple sclerosis, amyotrophic lateral sclerosis, dementia, or parkinsonism. Many patients facing these devastating diseases maintain hope for a curable diagnosis, such as Lyme disease; this optimism is bolstered by Internet resources supporting such notions. A critical point, however, is the highly focal geographic distribution of Lyme disease-none of the aforementioned neurologic diseases are unique to areas with high Lyme disease transmission. Furthermore, Lyme disease is readily distinguished from these conditions on clinical grounds. Patients with Lyme disease do not exhibit the white matter plaques seen on imaging of patients with multiple sclerosis, for example, and when patients with Lyme disease have oligoclonal bands in their cerebrospinal fluid, they are actually reactive against $B$ burgdorferi. Lyme disease does not produce the upper motor neuron signs seen in amyotrophic lateral sclerosis. ${ }^{4}$

While sometimes considered as an explanation for objective neuropathology, more often asked is if Lyme disease explains subjective neurocognitive dysfunction. Such inquiries likely stem from early reports of neurocognitive symptoms accompanying objective, inflammatory manifestations of Lyme disease, including Lyme arthritis. ${ }^{1}$ While such symptoms can be seen with Lyme disease, this association should not be taken to mean that all patients with subjective neurocognitive dysfunction have Lyme disease; such symptoms can also be found not only among patients with other infectious and non-infectious inflammatory conditions, but also among otherwise healthy persons. ${ }^{1}$ Even among patients with Lyme disease, the presence of subjective neurocognitive symptoms is more likely to reflect systemic inflammation than genuine central nervous system infection. This point was highlighted in a study of patients with erythema migrans, among whom the presence of symptoms such as headache, vertigo, paresthesias, and memory, concentration, or sleep disturbance did not predict the presence of cerebrospinal fluid (CSF) pleocytosis indicative of authentic central nervous system infection. ${ }^{5}$

If after careful consideration, neuroborreliosis is entertained as a diagnostic possibility, can laboratory testing help confirm or exclude the diagnosis? For patients with syndromes compatible with Lyme neuroborreliosis, such as seventh nerve palsy or aseptic meningitis, the positive predictive value of serologic testing is high, as is the negative predictive value of acute followed by convalescent testing. IgG immunoblots are particularly important, especially when considering the high prevalence of false-positive IgM immunoblotsperhaps the most common pitfall of Lyme diagnostics. In one representative series, more 
than $50 \%$ of patients with headaches and nearly $25 \%$ with neurocognitive symptoms thought potentially attributable to Lyme disease were due to something else, as a false-positive IgM immunoblot was the only test result suggesting $B$ burgdorferi infection. ${ }^{6}$ The high (27.5\%) prevalence of false-positive IgM immunoblots in this series is one of the primary reasons screening for Lyme disease is discouraged when the diagnosis is improbable. The IgM immunoblot is only useful for patients with illnesses of less than 4 weeks' duration that are compatible with early Lyme disease. After 4 weeks of illness, Lyme IgM immunoblots should be disregarded irrespective of their reported result.

The attribution of IgG immunoblot seropositivity to a patient's illness still requires clinical judgment; a positive IgG immunoblot is nondiagnostic without a compatible clinical syndrome. Low probability testing often lands patients in consultants' offices, creating challenges explaining results and countermanding the often patient-driven inclination to "just try antibiotics" that may lead to harmful drug adverse effects without chance of benefit. This approach may also delay arrival at the correct diagnosis and treatment.

In the event that the diagnosis of neuroborreliosis remains plausible but uncertainty remains, assessing the ratio of $B$ burgdorferi antibodies between CSF and blood can be useful. Isolated CSF antibody testing is discouraged, as correcting for blood antibody levels is essential to distinguish intrathecal antibody production from spillage into the CSF. A ratio of 1.3 or more is usually considered positive, but as with all antibody-based Lyme diagnostics, considering the clinical context is essential. Furthermore, elevated CSF antibody levels can persist even after adequate antibiotic therapy.

Patients with persistently positive serologic test results and ongoing symptoms can present a challenge, especially when a compelling alternative diagnosis has not been discovered. Unlike syphilis, where the rapid plasma reagin titer declines with adequate treatment, there is no test of cure for Lyme disease. Patients without new symptoms or findings should not be retested or retreated because seropositivity, including IgM, can persist for decades. For patients with a history of Lyme disease and persistent symptoms, the ineffectiveness of additional courses of antibiotic therapy in those previously treated should stay further antibiotics. $^{7}$

While $B$ burgdorferi infection can cause neurologic disease, familiar presentations far outnumber atypical manifestations. Consultants should base neuroborreliosis diagnoses on epidemiology, objective findings, and sound laboratory testing. When Lyme disease is deemed unlikely, educating patients and referring physicians alike will help avoid unnecessary antibiotic therapy and direct consideration of alternative diagnoses. While Lyme disease is no imposter, syphilis' title as the Great Imitator remains secure for now.

\section{Acknowledgments}

Funding/Support: Dr Lantos was supported by the National Center for Advancing Translational Sciences of the National Institutes of Health under award KL2TR001115. Dr Auwaerter was supported by the Sherrilyn and Ken Fisher Center for Environmental Infectious Diseases.

Role of the Sponsor: The funders had no role in the design and conduct of the study; collection, management, analysis, and interpretation of the data; preparation, review, or approval of the manuscript; and decision to submit the manuscript for publication. 


\section{References}

1. Halperin JJ. Nervous system Lyme disease: is there a controversy? Semin Neurol. 2011; 31(3):317324. [PubMed: 21964848]

2. Bakker R, Aarts MC, van der Heijden GJ, Rovers MM. No evidence for the diagnostic value of Borrelia serology in patients with sudden hearing loss. Otolaryngol Head Neck Surg. 2012; 146(4): 539-543. [PubMed: 22394551]

3. Sibony P, Halperin J, Coyle PK, Patel K. Reactive Lyme serology in optic neuritis. J Neuroophthalmol. 2005; 25(2):71-82. [PubMed: 15937426]

4. Galbussera A, Tremolizzo L, Isella V, et al. Lack of evidence for Borrelia burgdorferi seropositivity in Alzheimer disease. Alzheimer Dis Assoc Disord. 2008; 22(3):308. [PubMed: 18769174]

5. Ogrinc K, Lotrič-Furlan S, Maraspin V, et al. Suspected early Lyme neuroborreliosis in patients with erythema migrans. Clin Infect Dis. 2013; 57(4):501-509. [PubMed: 23667259]

6. Seriburi V, Ndukwe N, Chang Z, Cox ME, Wormser GP. High frequency of false positive IgM immunoblots for Borrelia burgdorferi in clinical practice. Clin Microbiol Infect. 2012; 18(12): 1236-1240. [PubMed: 22369185]

7. Klempner MS, Baker PJ, Shapiro ED, et al. Treatment trials for post-Lyme disease symptoms revisited. Am J Med. 2013; 126(8):665-669. [PubMed: 23764268] 